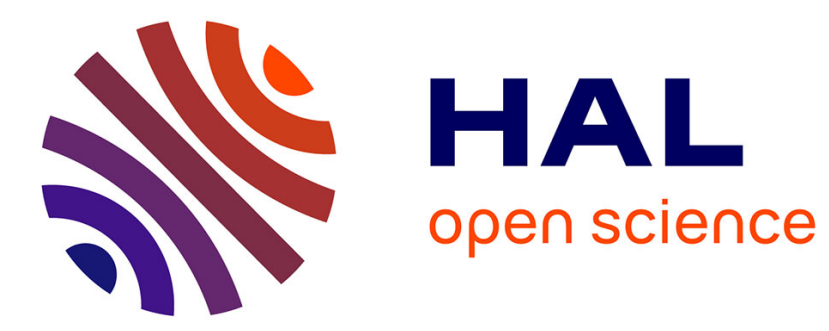

\title{
Measuring uncertainty assessment of an experimental device used to determine the thermo-optico-physical properties of translucent construction materials
}

Mélanie Delort, Ali Hamada Fakra, B Malet-Damour, Jean Claude C Gatina

\section{To cite this version:}

Mélanie Delort, Ali Hamada Fakra, B Malet-Damour, Jean Claude C Gatina. Measuring uncertainty assessment of an experimental device used to determine the thermo-optico-physical properties of translucent construction materials. Measurement Science and Technology, 2021, 10.1088/1361$6501 / \mathrm{ac} 407 \mathrm{~b}$. hal-03469898

\section{HAL Id: hal-03469898 \\ https://hal.science/hal-03469898}

Submitted on 8 Dec 2021

HAL is a multi-disciplinary open access archive for the deposit and dissemination of scientific research documents, whether they are published or not. The documents may come from teaching and research institutions in France or abroad, or from public or private research centers.
L'archive ouverte pluridisciplinaire HAL, est destinée au dépôt et à la diffusion de documents scientifiques de niveau recherche, publiés ou non, émanant des établissements d'enseignement et de recherche français ou étrangers, des laboratoires publics ou privés. 


\title{
Measuring uncertainty assessment of an experimental device used to determine the thermo-optico-physical properties of translucent construction materials
}

\author{
M. Delort ${ }^{1}$, D. A. H. Fakra ${ }^{2}$, B. Malet-Damour ${ }^{2}$, J. C. Gatina ${ }^{2} \ddagger$ \\ ${ }^{1}$ Building and Environment Science (S.B.E) department, University of la Réunion, \\ 117 Rue du General Ailleret, 97430 Le Tampon, REUNION \\ ${ }^{2}$ Physics and Mathematical Engineering for Energy and the Environment (PIMENT) \\ Laboratory, University of la Réunion, 117 Rue du General Ailleret, 97430 Le Tampon, \\ REUNION \\ E-mail: fakra@univ-reunion.fr
}

\begin{abstract}
Studying construction materials' thermo-optical properties (i.e., thermal conductivity, optical reflectance, optical transmittance, and optical absorbance) is essential for improving human comfort within a building. Typically, these properties are measured independently using specific equipment. The emerging of new innovative construction structures, such as translucent materials, makes the experimental characterization of these properties more challenging to observe. Recently, a new device, called MultiCoefMeter (McM), which rapidly and simultaneously measures all these properties, has been created. The study described in this article covers the calculation technique for estimating measurement uncertainties linked to morphology, the parts, and the physical formula of the experimental apparatus. The measurement uncertainty estimates are obtained from knowledge of the color of the system's walls, placement, and form of the McM components, placement of measurement sensors, and the application of measurement collection equipment. Therefore, a thorough calculation analysis was performed on the sub-systems. Calculations are divided into two categories: those based on mathematical tools and information given by the makers and those based on experimental observations obtained during reliability testing. These uncertainties originate from statistical tools, geometric tolerance of the system, comparison with standards, and the error propagation laws of the physical models link with the device. All these uncertainties were summed up and given a global value, no more than 5\%, conforming to the ASTM standard (E1225). Finally, a general method to quantify the measurement uncertainty value of any experimental device was proposed.
\end{abstract}

Keywords: Measurement, Optical, Thermal conductivity, Uncertainty.

$\ddagger$ Present address: SBE department, University of la Réunion, 117 Rue du General Ailleret, 97430 Le Tampon, REUNION 
Submitted to: Meas. Sci. Technol. 


\section{Introduction}

Numerous advancements have been achievied in the idea to conceive of new building materials. These materials are becoming more complicated and innovative. Examples of these materials include Phase Change Material (PCM) or translucent. These materials exhibit complex and difficult-to-measure thermo-optical-physical characteristics, such as optical absorption, reflectance, transmittance, and thermal conductivity. Knowledge of these characteristics may help enhance thermal and visual interior comfort. The instruments available for measuring these coefficients are prohibitively costly and measure only one variable at a time.

Additionally, the duration of the experiment is quite lengthy. For instance, it is essential to consider a half-hour time step on a conductivity meter to get more realistic and exploitable findings when measuring the thermal conductivity of construction material. The Multicoefmeter (McM) developed by Fakra and al. described in the article [1] may be used to determine the thermo-optical-physical coefficients of complex construction materials (see Figure 1). As with any other measuring instrument, the McM has measurement uncertainties. An uncertainty assessment calculation is required to ensure metrological traceability, accuracy, and reliability of measurement results. this analytical study based on mathematical tools must imperatively be carried out to ensure the reliability of measurements during an experiment [2]. Analytical computing tools, denoted GUM (Guide to the expression of Uncertainty in Measurement), capable to analyze the uncertainties of measuring instruments are available in the scientific litterature [3]. However, these techniques consider just one phenomenon at a time and not all of an experimental device's information. In other words, there is no universal method for calculating the uncertainty associated with a collection of sensors capable of concurrently measuring several physical variables. In the case of novel materials, the issue is magnified by the increased complexity of measurement [4],[5]. These investigations on the characterization of innovative materials include significant errors, which may affect the estimations of measurements on these building materials during studies of comfort in thermal and optical building studies [6], [7], [8], [9].

Previously, the McM system uncertainties were only determined experimentally from various materials that thermo-optical properties were known. It is critical to theoretically validate this uncertainty rate and provide a universal methodology for evaluating the global uncertainty of any comparable measurement system type.

Three main steps must be performed to do this assessment (see Figure 2):

- To begin, the uncertainties values associated with each measurement system component, for example, the McM, must be identified.

- Then, the uncertainties associated with each system's thermal and optical model are assessed using both the uncertainty propagation rule and global sensitivity analysis. 
- Finally, the total of previously calculated uncertainties yields the measurement system's overall uncertainty rate, for example, the McM in this present study.

\section{Brief description of the MulticoefMeter (McM)}

The thermo-optical properties of numerous construction materials samples were evaluated on the McM (see Figure1) whose dimensions are indicated in the table 1. Each sample was integrated into the specimen's holder (c) of the McM, between the two spheres (b), and in the laser path (a). The radiative flux measurements at positions (d), (e), and (f) is performed using the PIN micro photodiode described in [1]. These microsensors make it possible to determine the amount of laser radiation transmitted, absorbed, and reflected by the sample. The measuring device's relative errors are calculated using the measurement results from the standard samples tested on the McM. In addition, multiple experimental series (at least three identical measurements per specimen) were conducted and exploited to verify that the measurement results were repeatable. The McM differentiate two types of measurements:

(i) One for characterizing the specimen's optical properties: Due to the tiny size of the test specimens, steady-state conditions are reaching at last two minutes. The procedure consists of two phases. The first measurement (i.e., M1) is done before the laser is turned on (i.e., to account for the thermal effects of the environment outside the two spheres that influence the sensors). Then, a second measurement (i.e., M2) is taken (a few minutes later). These measurements reflect the global flow that comes from both within and outside the spheres identified by the sensors. The difference between these two fluxes (i.e., M2-M1) is the radiant flux generated only by the laser (i.e., a measurement without thermal or optical influence from the environment outside the two spheres).

(ii) The other measurement is for the specimen's thermal conductivity. The starting conditions required to achieve the thermal steady state for thermal conductivity tests depend on the investigated material. The item at study is positioning between two Peltier plates. The heat flux generated by the Peltier plates used to calculate this constant of thermal conductivity is sample-dependent. At the same time, the first plate warms the lower side of the sample, and the second cools the upper side. Thermocouples on either side of the component and between the Peltier plates and the specimen surfaces measure the flux. The difference between these temperatures lets us calculate the sample's thermal conductivity coefficient in a steady state from the Fourier law. 


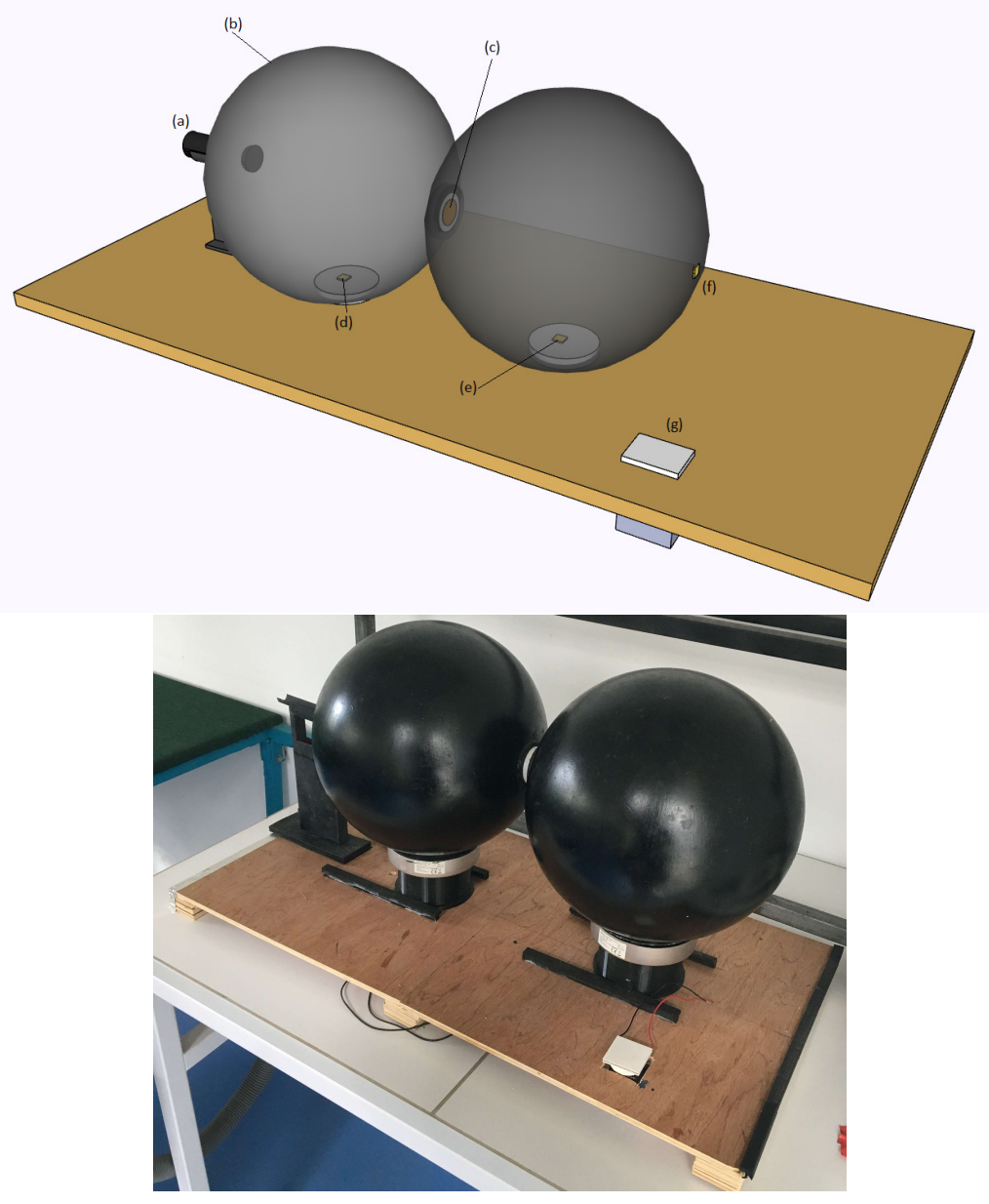

Figure 1. MulticoefMeter (McM): (a): Laser; (b): Integrating sphere; (c): Sample in its support; (g): Conductimeter; (d), (e) and (f): fluxmeters (i.e., PIN microphotodiodes) 


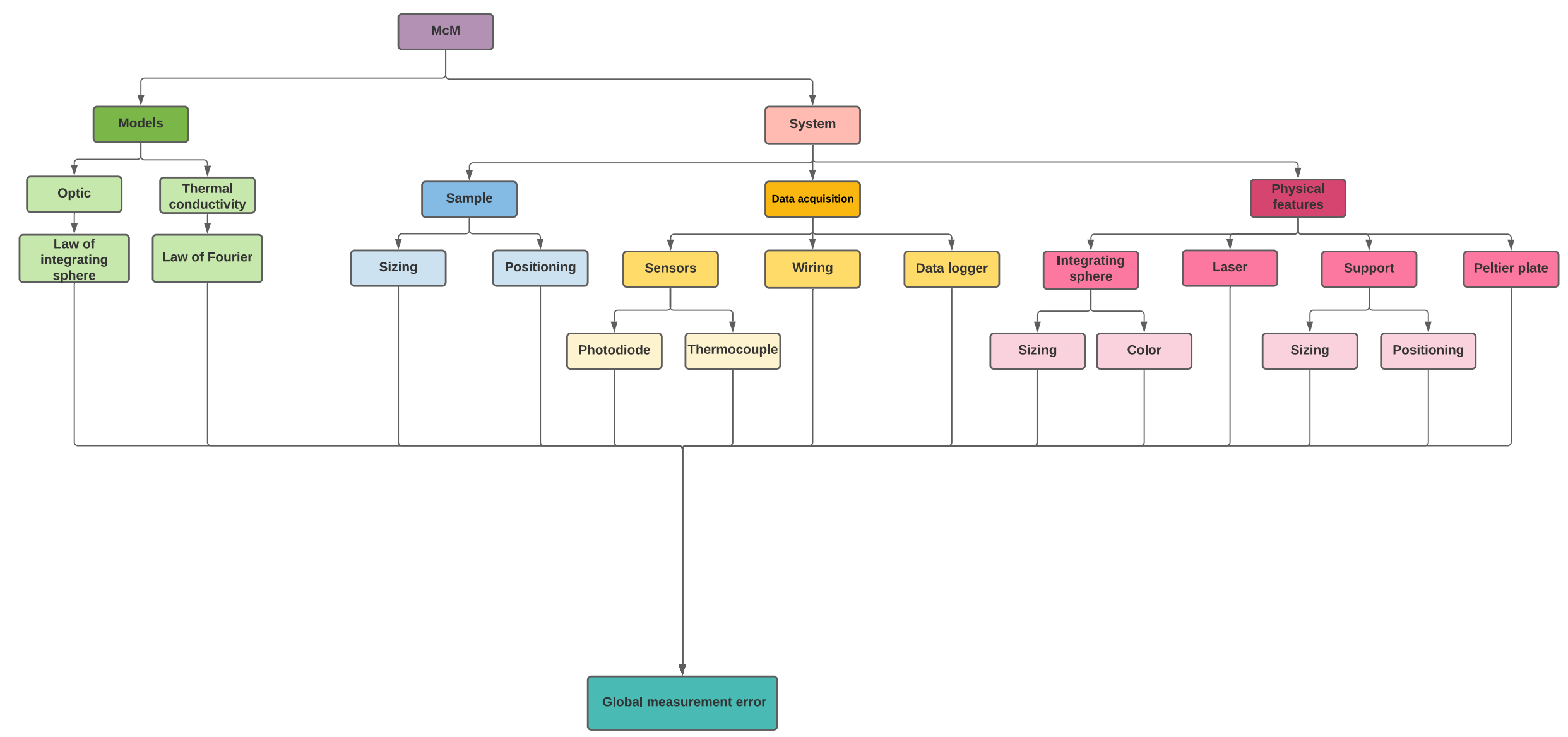

Figure 2. Uncertainty verification steps 
Table 1. Dimensions of the McM components.

\begin{tabular}{lll}
\hline Components & Physical variables & Values \\
\hline sample & $\begin{array}{l}\text { diameter } \\
\text { height }\end{array}$ & $35 \mathrm{~mm}$ \\
& $5 \mathrm{~mm}$ \\
\hline Integrating sphere & diameter & $0.30 \mathrm{~m}$ \\
\hline Specimen support & inner diameter & $35 \mathrm{~mm}$ \\
& outter diameter & $45 \mathrm{~mm}$ \\
\hline Laser & wavelength & $650 \mathrm{~nm}$ \\
& Dimensions & $20 \mathrm{~mm} \times 140 \mathrm{~mm}$ \\
\hline Peltier plate & Dimensions & $40 \mathrm{~mm} \times 40 \mathrm{~mm}$ X 3.8 mm \\
\hline
\end{tabular}

\section{Repeatability and reproductibility for measurement uncertainties of the $\mathrm{McM}$}

The uncertainty associated with type A is measured via a statistical study of a series of measurand values $X_{i}$ acquired under repeatability of the measuring (see [10] for more information). On the other hand, Type B uncertainties are associated with measurements for which statistical analysis is either impossible or useless. These uncertainties sum up in the reading obtained using a digital or graded instrument, a calibrated instrument, or another kind of equipment. Repeatability and reproducibility of the measuring device are determined only using type A and type B uncertainty (see reference [11]). These two complementary uncertainties combine to produce the system's total uncertainty.

\section{Uncertainties concerning the sample using in the McM}

Measurement uncertainty due to the sample may estimate by measuring its size and location in the sample holder. The two paragraphs that follow provide the mathematical techniques for quantifying these uncertainties.

\subsection{Calculating uncertainty with the Limiting distribution technique}

The limiting distribution [12] determines the uncertainty caused by random fluctuations in a finite set of $\mathrm{N}$ measures. The sample means distribution is typical, with no lower bounds and upper boundaries, implying that a maximum level of uncertainty cannot be determined. The standard deviation $\sigma[\bar{Y}]$ allows calculation of the width of the sample mean $(\bar{Y})$ distribution. As a result, one can only establish a standard uncertainty for random fluctuations. 
This standard uncertainty $\Delta Y$ owing to random fluctuations is considered to be equal to the standard deviation of the sample mean distribution:

$$
\Delta Y=\sigma[\bar{Y}]
$$

In other words:

$$
\Delta Y=\sqrt{\frac{1}{\mathrm{~N}(\mathrm{~N}-1)} \sum_{i=1}^{N}\left(Y_{i}-\bar{Y}\right)^{2}} \quad \text { where } \mathrm{N} \geq 2
$$

Finally, the relative uncertainty is :

$$
\varepsilon_{Y}=\frac{\Delta Y}{\bar{Y}} \times 100
$$

\subsection{Position of the Sample in the sample holder}

Geometric tolerances, as stated in [13], limit the permitted changes in the shape, orientation, or position of a component. Tolerances aid in the calculation of measurement uncertainties. The clearance between the sample and its support is primarily responsible for sample positioning issues. The concentricity between these two elements (represented in Figure 3) must be analyzed to determine it. Because they are assembled by hand, the appropriate tolerance class for this assembly are: H7h6. These tolerance classes provide information about the type of fit, the fit itself, and the potential communicated effort. The corresponding gaps for this fit, expressed in $\mu \mathrm{m}$ and stated for a diameter of $35 \mathrm{~mm}$, are indicated in Table 2 .

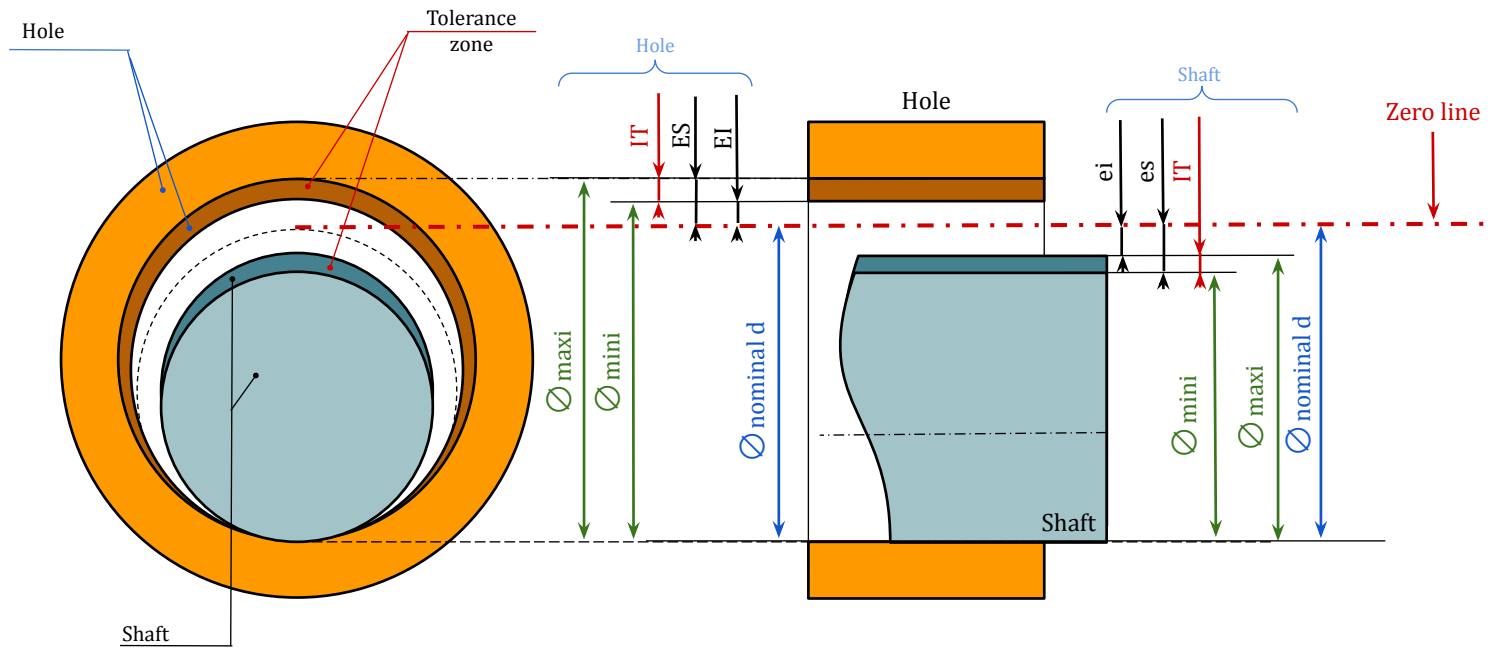

Figure 3. Principles of standardized adjustments ISO 286-1 [14]

Conforming to the standard [14], the maximum and minimum diameters of the two elements are determined with Table 3 and the measured diameter $d$. 
Table 2. Corresponding gap for the tolerance class H7h6.

\begin{tabular}{llll}
\hline & Shaft & Hole & \\
\hline & $\mathrm{h} 6$ & $\mathrm{H} 7$ & \\
\hline $\mathrm{es}$ & $\mathrm{ei}$ & $\mathrm{ES}$ & $\mathrm{EI}$ \\
\hline $0 \mu \mathrm{m}$ & $-16 \mu \mathrm{m}$ & $+25 \mu \mathrm{m}$ & $0 \mu \mathrm{m}$ \\
\hline
\end{tabular}

Table 3. Expressions of maximum and minimum diameters.

\begin{tabular}{lll}
\hline & Shaft & Hole \\
\hline$d_{\max }$ & $d+\mathrm{es}$ & $d+\mathrm{ES}$ \\
\hline$d_{\min }$ & $d+\mathrm{ei}$ & $d+\mathrm{EI}$ \\
\hline
\end{tabular}

These diameter values are used to indicate a maximum and minimum clearance $C_{\max }$ and $C_{\min }$. After that, the average clearance $\Delta C$ is determined as:

$$
\begin{aligned}
& C_{\text {max }}=d_{\text {max }_{\text {Hole }}}-d_{\text {min }_{\text {Shaft }}} \\
& C_{\text {min }}=d_{\text {max }_{\text {Shaft }}}-d_{\text {min }_{\text {Hole }}} \\
& \Delta C=\frac{C_{\text {max }}-C_{\text {min }}}{2}
\end{aligned}
$$

This final result is used for the error calculation described in the preceding section:

$$
\varepsilon_{C}=\frac{\Delta C}{d} \times 100
$$

\section{McM data acquisition chain}

This section outlines the uncertainty around each McM part (i.e., the sample, the data acquisition chain, and the thermo-optical and physical properties of construction materials of the McM system). Each method for estimating the uncertainty associated with these McM components is described in depth.

Sensors, wiring, and a central data acquisition unit constitute the data acquisition chain. The central data acquisition unit transmits and combines all data gathered by the McM. Naturally, each of these components impacts the McM's measurement and, therefore, on its total uncertainty.

\subsection{Sensors errors:}

The McM is equipped with two types of sensors to identify the thermo-optical and physical properties of the sample. Fluxmeters are used to measure thermal flux, while 
thermocouples are employed to quantify the temperature.

5.1.1. Global measurement error for thermocouples: Calibration testing is the best method for determining the inaccuracies associated with each thermocouple utilized. The Figure $4, S_{\text {ref }}$ show the principle. The reference thermocouple $S_{r e f}$ with $T_{r e f}$ temperature comparing with a thermocouple $S_{x}$ to be tested with a temperature $T_{x}$. These two thermocouples are placed in a controlled temperature environment $T_{\text {env }}$.

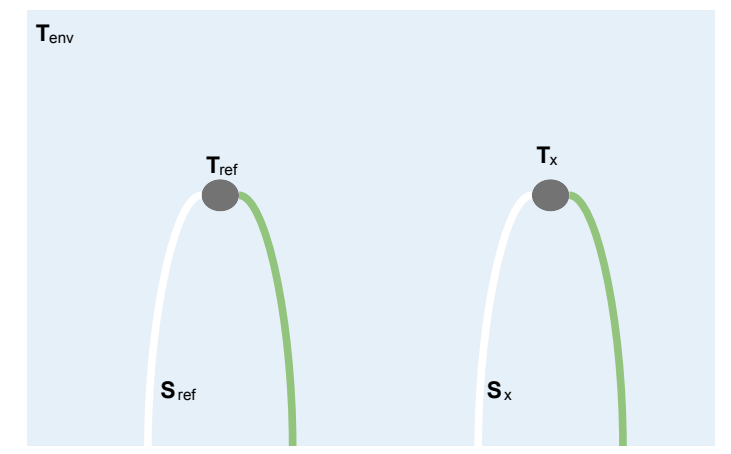

Figure 4. Thermocouple $S_{x}$ calibration via comparison with a standard $S_{r e f}$

Using the conversion table of the standard [15], this thermocouple's voltage measured by a voltmeter is then converted into kelvin $(\mathrm{K})$ or in Celsius degree $\left({ }^{\circ} \mathrm{C}\right)$. After converting the voltage of each thermocouple, the temperature difference $\Delta T_{\text {thermocouple }}$ is calculated from the equation (5.1).

$$
\Delta T_{\text {thermocouple }}=\left|T_{\text {ref }}-T_{x}\right|
$$

Equation (5.1) is utilized in equation (5.2) to get the sensor's measurement uncertainty.

$$
\varepsilon_{T}=\frac{\Delta T_{\text {thermocouple }}}{T_{\text {ref }}} \times 100
$$

These errors are compared to the applicable standard or specific tolerance limits indicated in Standard [15] to verify if they are acceptable.

5.1.2. Measurement and positioning uncertainties for fluxmeters. It is laborious to measure heat flow with traditional sensors on a curvilinear surface (i.e., within an integrating sphere). An innovative solution proposes installing microscopic photodiodes capable of performing the same function as a traditional fluxmeter (see the article of Fakra et al. [1]). A reference fluxmeter must calibrate these sensors with the procedure described in the article [1]. The standard fluxmeter and the photodiodes are placed at equal distance from the source in a box (see Figure 5) at $30 \mathrm{~cm}$ height. The inside of the box must be a highly reflective surface (i.e., painted white, for example). The 
optical source is a laser installed in the upper of the box closed during the calibration. The laser is turning on, and the distance between it and the two sensors in the white box is adjusted to get a range of measured flux values that will calibrate the sensors. A calibration factor $k$ is then determined by dividing the value measured by the photodiode with the values measured by the fluxmeter. This factor serves to convert the tension in $m V$ of the photodiode into $W \cdot m^{-2}$. The method for calculating $k$ is presented in detail in the article [1] and the measured values are provided in the technical note [16]. The method use to determinate The photodiodes' measurements are influenced by their self uncertainty and their positioning in the system.

(i) Measurement error: The measurement error of the photodiode may then be estimated. Figure 5 shows an example positioning of a calibrated reference fluxmeter $F$ measuring a flux density $V_{\text {ref }}$ in $W \cdot m^{-2}$ and a photodiode A measuring a voltage $V_{A}$ putting in the same box used for the calibration.

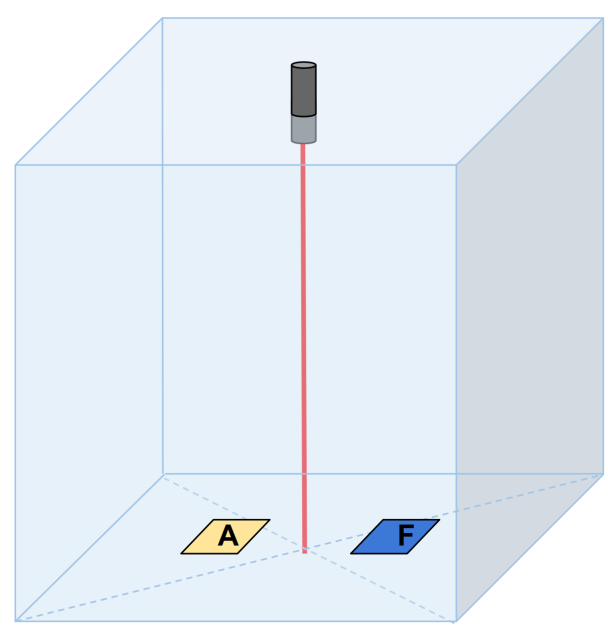

Figure 5. Photodiode difference measurement method

To convert $V_{A}$ to a flux density, use the previously determined calibration factor $k$ as:

$$
\Delta V_{A}^{\prime}=k \times V_{A}
$$

Then, the difference $\Delta V$ between the two measured values is:

$$
\Delta V=\left|V_{\text {ref }}-V_{A}^{\prime}\right|
$$

This value is computed in the following equation to produce a measurement uncertainty as:

$$
\varepsilon_{V}=\frac{\Delta V}{V_{\text {ref }}} \times 100
$$


(ii) Positioning uncertainties: The photodiode's misplacement is caused by (a) its distance from the sample (b) its inclination relative to the surface of the integrating sphere. The methods of the subsection 4.1 and 4.2 are used to determine these uncertainties. In resume, Two situations arise:

(a) The sensor location from the sample must be rigorously known to ensure high precision of the reflectance, absorbance, or transmittance coefficients. The fluxmeters' locations (i.e., A, B, and C) and the distance of these fluxmeters from the sample are shown in Figure 6 (see [17] for more information about positioning sensors). Repeated measurements of the distances (i.e., $x_{A}, x_{B}$ and $x_{C}$ ) between the fluxmeters and the sample allowed for establishing the uncertainties associated with the sensor positioning. The calculation method used is identical to that described in the subsection 4.1 .

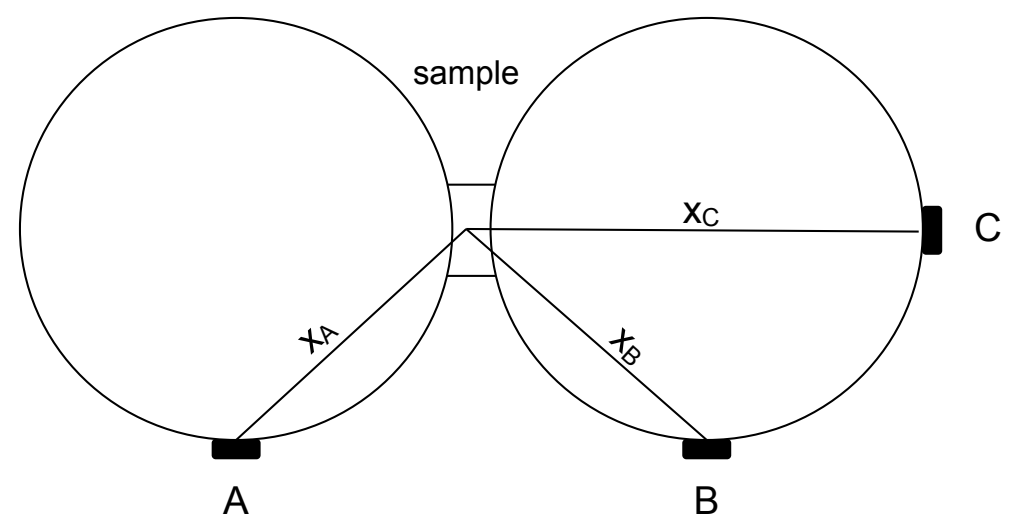

Figure 6. Distance between Sensors and the sample in the integrating spheres

(b) Geometric tolerances rules enable the curvilinear surfaces to be assimilated into straight lines when these are infinitely small, which is the case when the micro photodiode is inserted on a tiny piece of the interior surface of one of the much larger spheres used in the McM. We then have perfect parallelism between the surface of the photodiode and that of the sphere according to the AFNOR E165321 standard given by [1].

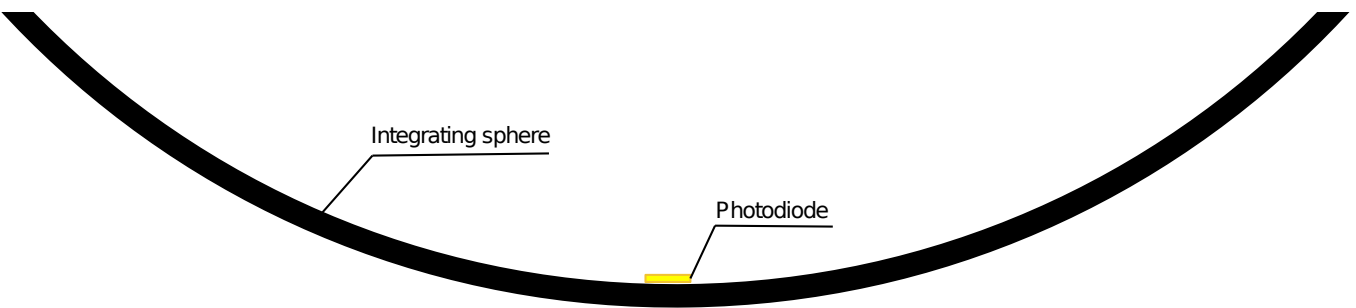

Figure 7. Parallelism between the photodiode and the sphere surface 
This tolerance zone $t$ or form tolerance represented in figure 8 is defined by planarity which is defined in the standard [18], and is determined by the distance between two parallel planes (i.e., equal to the thickness of the photodiode $h$ in our study case).

After many measurements of $h$, the maximum, minimum, and average values are collected using the method of the subsection 4.1. Finally, the tolerance value $t$ is determinate from the equation below :

$$
t=h+2 \varepsilon_{h}
$$

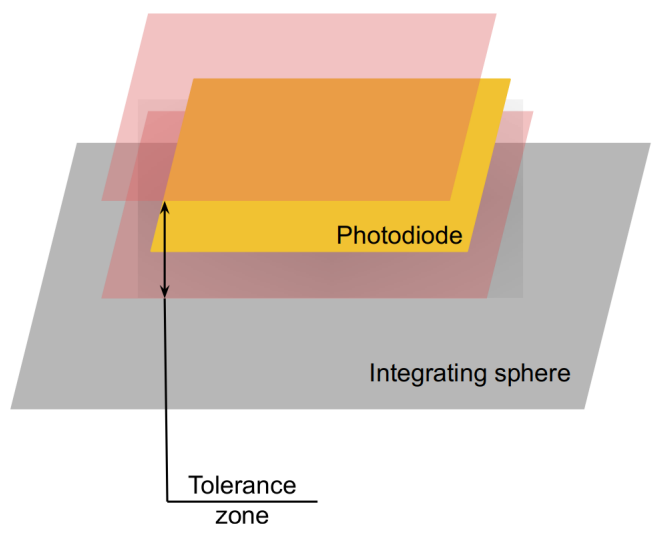

Figure 8. Tolerance zone between the photodiode and the sphere surface

5.1.3. Wiring uncertainty: The sensor's signal transmission wires are assimilated with linear resistances. Each of these resistances is measured $N$ times then analyzed according to the procedure described in the subsection 4.1 to ascertain the measurement disturbances caused by the cables.

5.1.4. Data logger uncertainty. For the McM to record the electrical signal values provided by the sensors, a data logger is needed. Electrical signal calibrators can be used for the test (i.e., a voltage generator in most cases). Consider a calibrator that outputs an electrical signal $E_{r e f}$ at a data logger showing the signal value $E_{\text {data }}$. The following formula is used to calculate the uncertainty values of the data logger:

$$
\varepsilon_{E}=\frac{\left|E_{\text {ref }}-E_{\text {data }}\right|}{E_{\text {ref }}} \times 100
$$

The accuracy of a data logger is also included in the manufacturer's data sheet. 


\section{McM physical features}

The integrating spheres, laser, specimen support, and Peltier plates are the materials that constitute the McM's. All of these elements indirectly affect the device's measurements. As a result, it is essential to investigate their uncertainty.

\subsection{Integrating spheres uncertainties.}

The integrating sphere's surface and color are critical to the measurement. The vast majority of optical experiments are based on these properties. Their uncertainties must be quantified.

6.1.1. Surface of the integrating sphere. Many factors, such as reflectance and transmittance, are affected by the internal surfaces of the integrating spheres and indirectly by their diameters $D_{\text {sphere } 1}$ and $D_{\text {sphere } 2}$. The uncertainty due to measuring the diameter of the integrating sphere is determined the same in the subsection 4.1.

6.1.2. Color of the integrating wall. To make the integrating sphere Lambertian, the inner surface of the system has been painted matt white inside and matt black outside (see picture Figure 9). However, the inner paint using must respect some requirements, as pointed out in the article [1]:
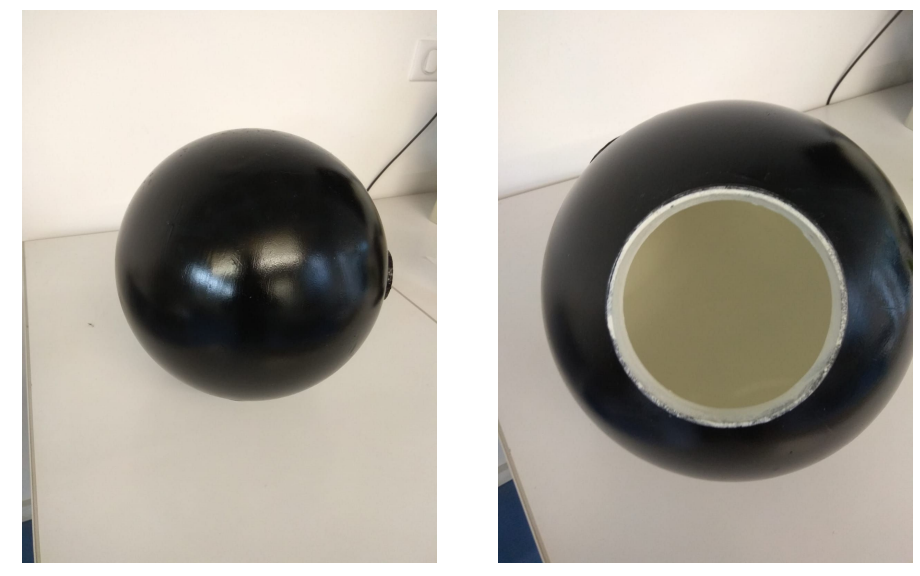

Figure 9. Painted integrating spheres: white inside and black outside 
- First, it must have diffuse reflectance characteristics and exhibit a high and constant reflectance over a broad range of wavelengths from ultraviolet to infrared.

- Secondly, this white paint must be resistant to high-intensity optical radiation and meet requirements for chemical inertness.

This paint will have a significant impact on the incident radiant flux error. The type of paint has a reflectance coefficient $\rho$ indicated in the manufacturer's technical notice. This coefficient is used to calculate the paint's inaccuracy as :

$$
\varepsilon_{\rho}=100 \%-(\rho \times 100)
$$

6.1.3. Laser uncertainty. A thermal flux emission source is utilized to calculate the sample's thermo-optical characteristics. This source is generally monochromatic and coherent, such as a laser. The laser uncertainty might be found in the manufacturer's device's technical notice.

6.1.4. Specimen support uncertainties. The specimen support holds the sample in the integrating spheres. To precisely place the sample, the errors caused by position and sizing of the specimen support must be assessed same in the subsections 4.1 and 4.2.

(i) Size of the support: The sample support is characterize by an outer diameter $d_{\text {outter }}$, an inner diameter $d_{\text {inner }}$ and a height $e$ shown in Figure 10. Only the diameters are considered for the uncertainty assessment. The uncertainty on these sizes is calculated as in the subsection 4.1.
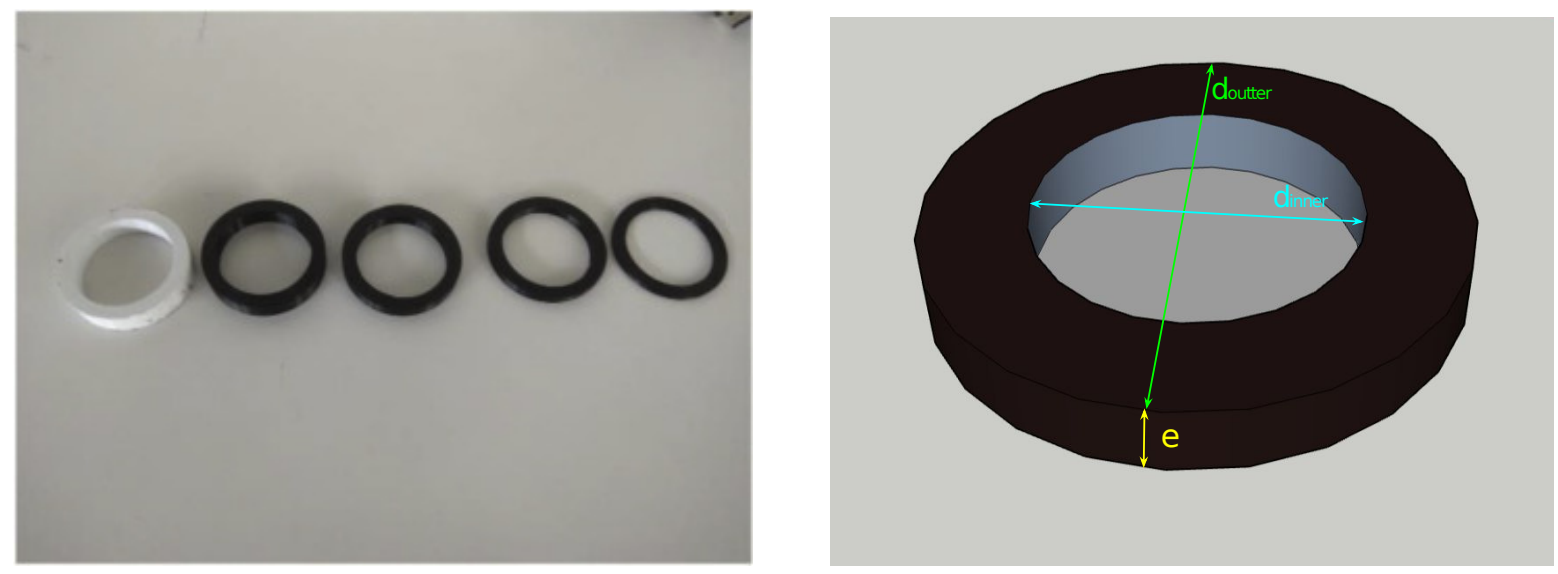

Figure 10. Specimen support form 
(ii) Position of the specimen support in the system: The support is positioned between the integrating spheres as in Figure 11. The spacing between the sample holder and the sphere contributes to the errors due to the placement. The positioning error is determined by examining the concentricity applied to the two cylinders orifices of the two integrating spheres on the sample holder's outer diameters. In this case, the method to calculate the uncertainties as described in the subsection 4.2.

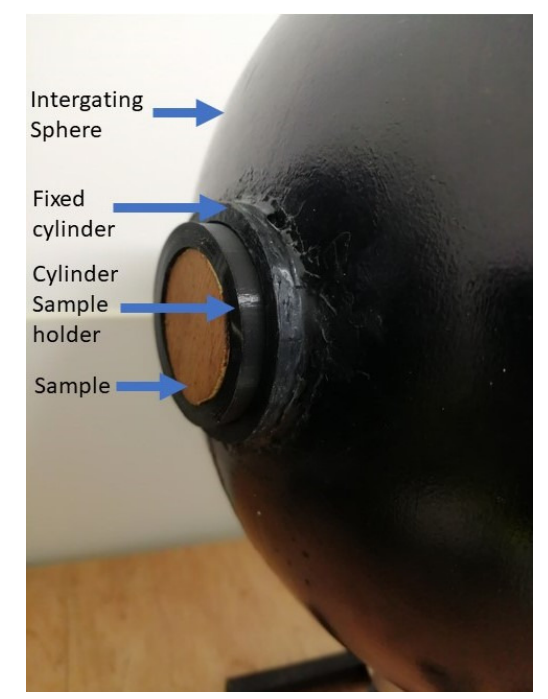

Figure 11. Contact between sample, cylinder sample holder fix cylinder and opening spheres

6.1.5. Peltier plate error. Peltier plate can generate a voltage if the two sides of the plate are in contact with two different heat sources (one hot and one cold). It is also possible to produce two heat sources (one hot et another cold) on either side of the Peltier plate if a continuous voltage circulates in the Peltier plate. The direction of current passing determines heat flux orientation through the plate. The two phenomena (i.e., voltage production from a heat transfer or the heater production from a voltage circulation in the Peltier plate) can be considered reversible. These Peltier plates are used as both hot and cold thermal sources in the McM during experimental measurement to determine the thermal conductivity of materials. The temperature gradient between these two heat sources from the same plate enables the thermal measurement error caused by this Peltier plate to be estimated. The experiment test then consists in considering a Peltier plate $P_{p}$ and two calibrated thermocouples $S_{1}$ and $S_{2}$ having corresponding temperatures $T_{1}$ and $T_{2}$, as shown in Figure 12 . To calculate the inaccuracy of heat transfer due to temperature differences of each surface plate, these measurements will be repeated at least five times, with the power range ranging from $0 \mathrm{~W}$ to the maximum power given in the manufacturer's device's technical 
notice. It is possible to find a linear right between this power and the thermal gradient $\Delta_{T_{r e f}}$. Equation linking point clouds measuring power provided by Peltier Plate and temperature gradient shows multiplier coefficient $\pi_{1,2}$ used to transform power delivered by Peltier Plate $P$ into temperature gradient. In the McM utilization, this multiplication coefficient may consider as an evaluating value for calculating the Peltier plate error.

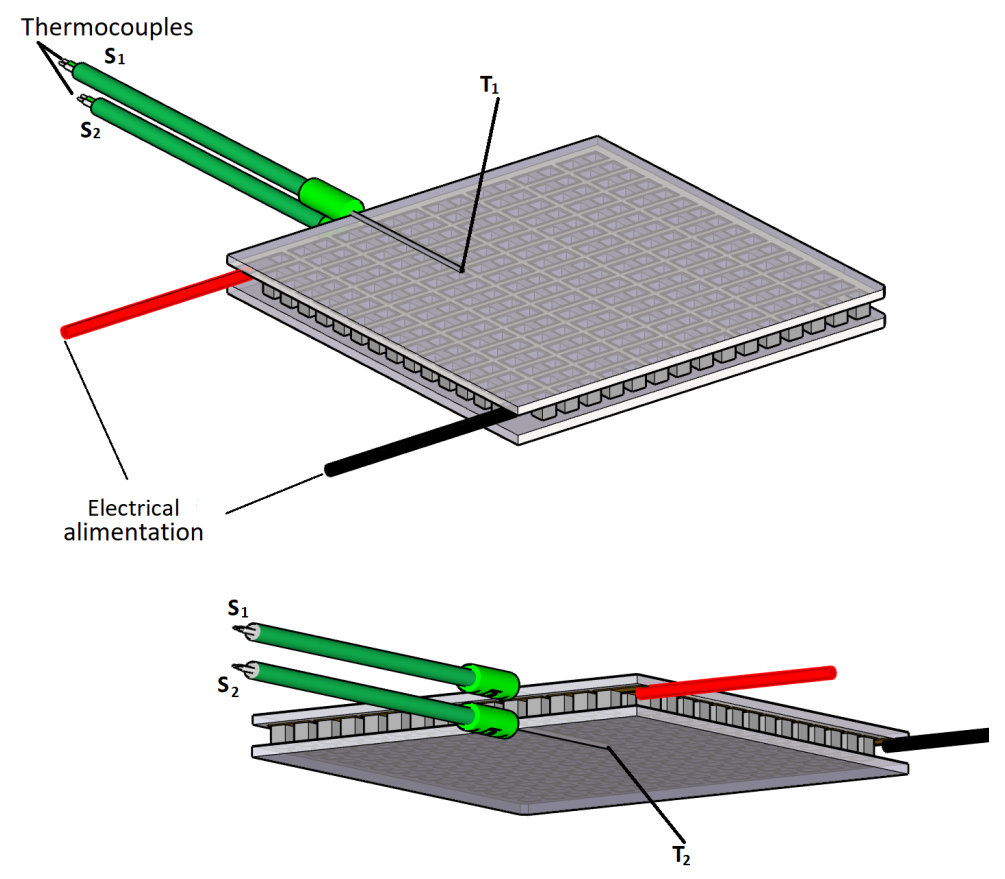

Figure 12. Two thermocouples on either side of the Peltier plate to measure surface temperature of the system

The temperature difference $\Delta_{T_{12}}$ between the two surfaces is computed as:

$$
\Delta_{T_{12}}=\left|T_{2}-T_{1}\right|
$$

Then, we obtain the next equation $\left(\Delta_{T}\right.$ must have the same unit):

$$
\varepsilon_{T_{12}}=\frac{\Delta_{T_{r e f}}-\Delta_{T_{12}}}{\Delta_{T_{\text {ref }}}} \times 100
$$

$\pi_{1,2}$ is acquired from the Peltier plate's technical note or by following a calibration method (see [19]) from the equation:

$$
\Delta_{T_{12}}=\pi_{1,2} \times P
$$




\section{General method for calculating the uncertainty of models used in the $\mathrm{McM}$}

The McM device's empirical models were proposed using a systems approach. This method is based on the system's inputs, outputs, and parameters (see Figure 13). These models describe the relationship between the system and its immediate surroundings and the existing relationship between the system's subsystems. The outputs values uncertainties are established thanks to the uncertainties associated with inputs values. The parameter value uncertainty is considered negligible.

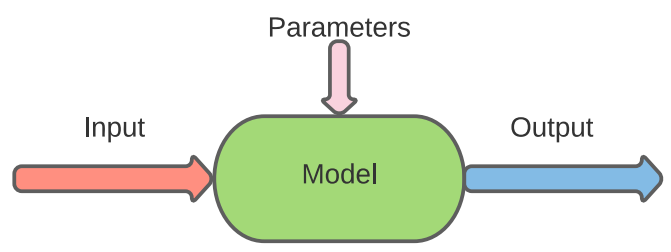

Figure 13. Model composition

Since the $M$ models are constructed via a series of compound functions (sum, difference, product, quotient, square, and power), it is possible to use the uncertainty propagation law as shown in [10]. Each term in the equation $X_{i}$ is created independently, and their associated uncertainties $\Delta X$ are known. In this instance, the covariance problem does not arise, and the law of uncertainty propagation becomes:

$$
u_{c}^{2}(M)=\sum_{i=1}^{N}\left(\frac{\partial M}{\partial X_{i}}\right) u^{2}\left(X_{i}\right)
$$

Conforming to the article [1], it is possible to write the variance as $u^{2}(x)=\left(\frac{s(x)}{\sqrt{N}}\right)^{2}$. Moreover, $\sigma(x)=\frac{s(x)}{\sqrt{N}}$, hence $u^{2}(x)=\sigma^{2}[x]$. As stated in the section 4.1, an existing relation between $\Delta x$ and $\sigma[x]$ leads to another relation: $u^{2}(x)=(\Delta x)^{2}$ and thus:

$$
u_{c}^{2}(M)=\sum_{i=1}^{N}\left(\frac{\partial M}{\partial X_{i}}\right)^{2} \sigma^{2}\left(X_{i}\right)
$$

Finally, the model uncertainty is equal to $\sqrt{u_{c}^{2}(f)}=\Delta M$ :

$$
\varepsilon_{M}=\frac{\Delta M}{\bar{M}} \times 100
$$

Additionally, the optical models established in the article [1] result in:

$$
1=\rho+\tau_{\text {diffuse }}+\tau_{\text {coherent }}+\alpha
$$

Where: 


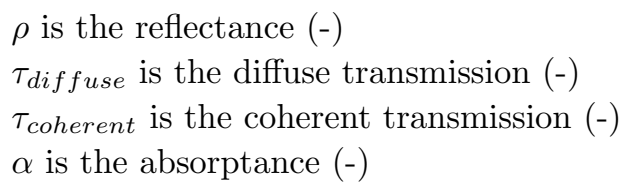

The uncertainties associated with $r h o$, tau, and alpha are defined by the uncertainty propagation rule described in [12]. Several parameters may influence them. To ensure the parameters we need are considered in the linear model estimation, we need to use the statistical tool of R.A. Fisher to build an experiment design (See reference [20] for more details). Let's consider a value $\mathrm{X}=\left(X_{1}, \ldots, X_{d}\right)$ be a d-factor vector. A factor is typically a criterion for selecting between two options. As a result, it is defined as a two-level factor. A complete factorial design with two levels necessitates $2^{d}$ tests. Once the matrix $\mathrm{X}$ is obtained, the matrix $\mathrm{Y}$ can be written as follows: $Y=X A$. The method of least squares is used to determine the coefficient matrix $A$ as:

$$
A=\frac{X^{t} Y}{2^{d}}
$$

If the factor $X_{i}$ accepts $n_{i}$ values, a complete factorial design will necessitate $n_{1} \times n_{2} \times$ $\ldots \times n_{d}$ tests.

A complete 2-level factorial design is built on a following form model:

$$
Y=a_{0}+\sum_{i=1}^{d} a_{i} X_{i}+\sum_{j>i}^{d} a_{i j} X_{i} X_{j}+\sum_{k>j}^{d} a_{i j k} X_{i} X_{j} X_{k}+a_{1 \ldots d} X_{1} X_{2} \ldots X_{d}
$$

Where:

$$
\begin{aligned}
& a_{0} \text { is the mean of the responses } \\
& a_{i} \text { is the principal effect of a factor } \\
& a_{i j}, a_{i j k} \text { are the interaction effects of factors }
\end{aligned}
$$

The law of uncertainty propagation may be used after establishing the linear model (i.e., Equation (6.6)).

\section{Global uncertainty of the McM}

The overall uncertainty is the total of the uncertainties of the McM system's components and models. Six measurements were taken to provide a precise uncertainty estimate of each system component and physical characteristic that required statistical analysis. Table 4 summarizes the uncertainty related to the McM components, models associated and the protocols or measurement device used.

The determination of the models' uncertainties was calculated with the following equations subjected to the experimental design:

$$
\rho=\frac{\phi_{\text {sphere }}}{\phi_{i}}\left[1-\rho_{w}(1-f)\right]
$$


Table 4. Total McM uncertainty.

\begin{tabular}{|c|c|c|c|c|}
\hline McM components & Nature & Equation used & Uncertainty (\%) & Comments \\
\hline \multirow[t]{2}{*}{ Sample } & Sizing & (4.2) and (4.3) & 0.138 & Sample: Iron \\
\hline & Positioning & (4.5) and (4.6) & 0.12 & Measurement device: Vernier caliper \\
\hline Thermocouple & Measurement & (5.1) and (5.2) & 0.2 & $\begin{array}{l}\text { Thermocouple: type K thermocouple } \\
\text { Protocol: } 5.1 .1\end{array}$ \\
\hline \multirow[t]{3}{*}{ Photodiode } & Measurement & $(5.4)$ and $(5.5)$ & 0.16 & $\begin{array}{l}\text { Photodiode: BPW34 Silicon PIN } \\
\text { Protocol: } 5.1 .2\end{array}$ \\
\hline & Distance & (4.2) and (4.3) & 0.048 & Measurement device: Measuring tape \\
\hline & Inclination & $(4.2)$ and (4.3) & 0.181 & Measurement device: Vernier caliper \\
\hline \multirow[t]{2}{*}{ Wiring } & Signal transmission & (4.2) and (4.3) & 0.53 & Jumper Wire \\
\hline & & & & Measurement device: Voltmeter \\
\hline \multirow[t]{2}{*}{ Data logger } & Data acquisition & $(5.7)$ & 0.1 & Data logger CR10X \\
\hline & & & & Uncertainty given by the manufacturer \\
\hline \multirow[t]{2}{*}{ Integrating sphere } & Sizing & (4.2) and (4.3) & 0.024 & Measurement device: Measuring tape \\
\hline & Color & $(6.1)$ & 1 & Uncertainty given by the manufacturer \\
\hline \multirow[t]{2}{*}{ Laser } & Optical source & - & 0.5 & Wavelengths: $650 \mathrm{~nm}$ \\
\hline & & & & Uncertainty given by the manufacturer \\
\hline \multirow[t]{2}{*}{ Specimen support } & Sizing & (4.2) and (4.3) & 0.066 & Built with a $3 \mathrm{D}$ printer \\
\hline & Positioning & $(4.5)$ and (4.6) & 0.09 & Measurement device: Vernier caliper \\
\hline \multirow[t]{2}{*}{ Peltier plate } & Thermal source & (6.2) and (6.3) & 0.7 & Peltier plate: TEC1-12706 \\
\hline & & & & Protocol: 6.1 .5 \\
\hline Total uncertainty & & & 3.857 & \\
\hline
\end{tabular}

Table 5. Models used in the McM.

\begin{tabular}{llll}
\hline Model & Output & Input & Parameter \\
\hline$\rho=\frac{\phi_{\text {sphere }}}{\phi_{i}}\left[1-\rho_{w}(1-f)\right]$ & $\rho$ & $\phi_{\text {sphere }}, \phi_{i}$ & $f, \rho_{w}$ \\
$\lambda=\frac{\varphi}{A_{x}} \frac{\Delta_{T}}{d_{x}}$ & $\lambda$ & $\varphi, \Delta_{T}$ & $A_{x}, d_{x}$ \\
$\tau=\frac{\phi_{\tau}}{\phi_{i}}$ & $\tau$ & $\phi_{i}, \phi_{\tau}$ & - \\
$\alpha=1-\rho-\tau$ & $\alpha$ & $\tau, \rho$ & - \\
\hline
\end{tabular}

$$
\lambda=\frac{\varphi}{A_{x}} \frac{\Delta_{T}}{d_{x}}
$$

The obtained linear models are:

$$
\begin{aligned}
& \rho=\rho_{0}+0.375 \phi_{i} \\
& k=k_{0}+1.013 \Delta_{T}
\end{aligned}
$$

where $\rho_{0}$ and $k_{0}$ are the mean value of $\rho$ and $k$

The coefficients $\phi_{\text {sphere }}$ and $\varphi$ are no longer considered in the newly developed linear models (i.e., Equations (7.3) and (7.4)) since their impact on the experimental design remains negligible. The inputs variables, outputs variables, and parameters of 
Table 6. Total models uncertainty.

\begin{tabular}{lll}
\hline Model & Variables & Uncertainty $(\%)$ \\
\hline$\rho$ & $\phi_{\text {sphere }}$ & 0.12 \\
& $\phi_{i}$ & \\
& $\rho_{\omega}$ & \\
& $\mathrm{f}$ & \\
\hline$k$ & $\Delta_{T}$ & 0.079 \\
& $A_{x}$ & \\
& $d_{x}$ & \\
& $\varphi$ & \\
\hline$\tau$ & $\phi_{\tau}$ & 0.161 \\
& $\phi_{i}$ & \\
\hline$\alpha$ & $\tau$ & 0.161 \\
\hline Total uncertainty & & \\
\hline
\end{tabular}

the thermo-optical models used in the McM are listed in Table 5. Table 6 shows the findings of the uncertainty analysis performed on the McM's output variables.

The total of the uncertainties given by Tables 3 and 5 represent the McM's theoretical overall uncertainty. This theoretical value $\varepsilon_{M c M}$ is $4.4 \%$ and is below the suggested ASTM E1225 cutoff uncertainty value (i.e., 5\%) for such a measuring device. The article [1] indicates that the maximum value obtained for the McM's experimental uncertainty is around $5.88 \%$ in the case of measuring a wood sample, for example. However, the system's theoretical estimate (i.e., 4.4\%) is slightly different. The discrepancy between theoretical and experimental measurement uncertainty of $\varepsilon_{M c M}$ is attributable to the fact that theoretical calculations do not account for the wear and tear of the McM device over time, unlike experimental estimation. In addition, accidental errors in the experimentally measured uncertainties due to equipment user handling may also create this discrepancy in uncertainty difference.

\section{Generalization of the method}

The flowchart in Figure 14 illustrates the basic procedure for calculating a measurement device's uncertainty. The approach takes all preceding techniques to show the components of a measuring system. Thus, the theoretical uncertainties calculation considers the physical properties of system components, related models, and the data collection chain (i.e., acquisition unit, wires, and sensors). 


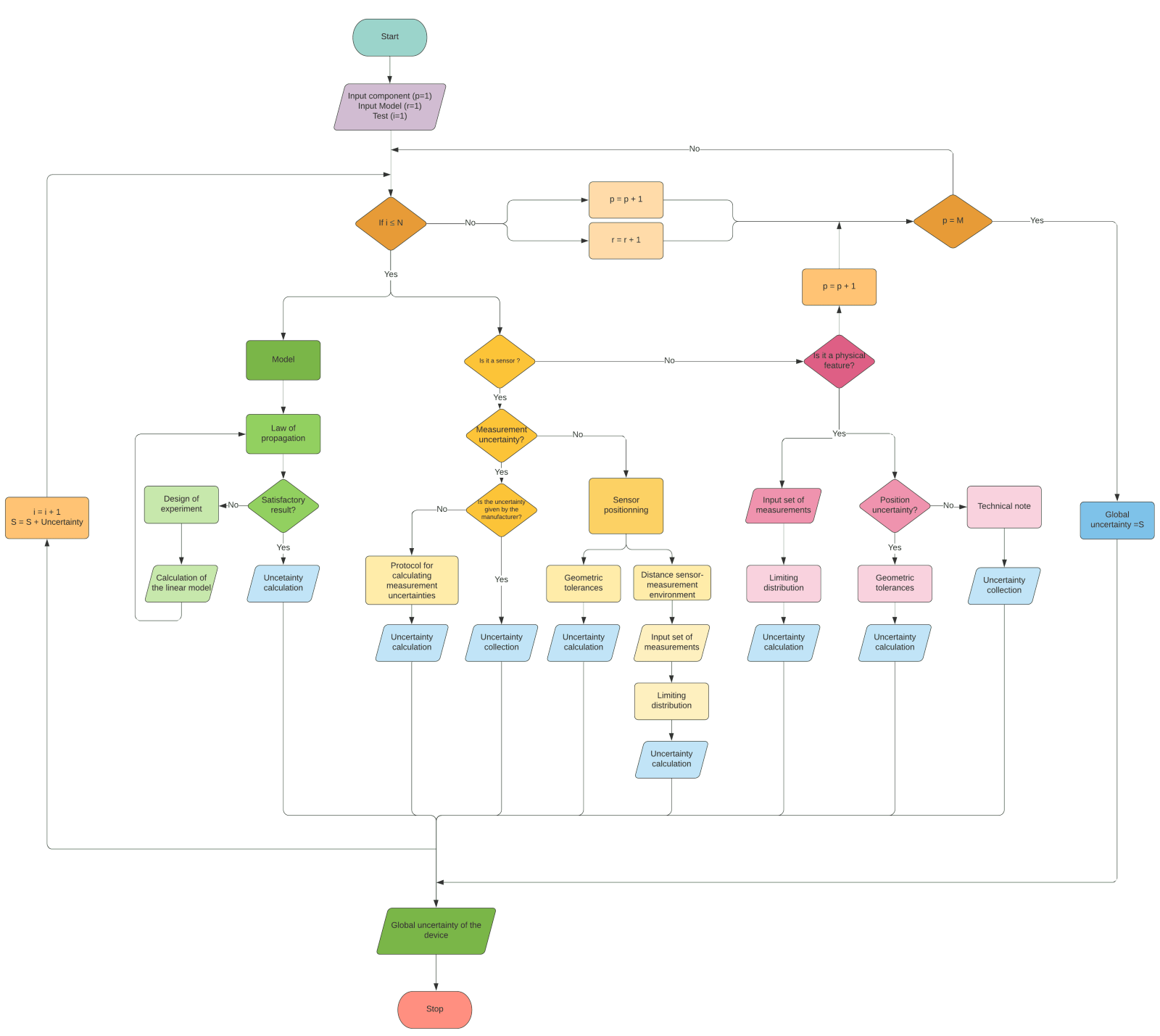

Figure 14. General method to determine a measurement device uncertainty 


\section{Conclusion}

Due to significant advancements in civil engineering, complex materials, such as transparent materials, have developed. However, determining their characteristics using currently available measurement equipment is challenging. The MulticoefMeter, or McM, was designed for this purpose. The devices are a cheap and straightforward tool for determining the thermo-optical-physical characteristics of building materials. The optical reflectance, optical transmittance, optical absorbance, and heat conductivity are the thermo-optical characteristics that the McM may measure.

Previously, experimental research was performed to estimate the measurement uncertainties of the McM. Further theoretical analysis of this measurement error is suggested here to verify the device's reliability degree. The presented theoretical laws estimate the uncertainties associated with the color, location, and form of the McM components that interfere with the measurements and the measurement acquisition equipment (i.e., the data logger, sensors, and wires). Concerning the uncertainties related to the physical most complicated models of the McM, the calculation was realized using a mix of the uncertainty propagation law and the system's component morphology. The resulting uncertainty of the $\mathrm{McM}$ is $4.4 \%$. This value remains acceptable to the recommendations of ASTM E1225. Finally, a general method to estimate the uncertainty of measurement devices has been proposed. This technique entails applying theoretical approaches to measuring equipment by examining each component to calculate the related uncertainty percent. 


\section{References}

[1] D. A. H. Fakra, B.R. Alfonso José, N. Mohammad Murad, A. Ny Aina Randriantsoa, and J. C. Gatina. A new affordable and quick experimental device for measuring the thermo-optical properties of translucent construction materials. Journal of Building Engineering, 32:101708, 2020 .

[2] Jailton Carreteiro Damasceno and Paulo R.G. Couto. Methods for Evaluation of Measurement Uncertainty. Metrology. Anil, 2018.

[3] JCGM. Guide to the expression of uncertainty in measurement — part 6: Developing and using measurement models. Technical report, Joint committee for guides in metrology, 2020.

[4] Hans-Rudolf Schalcher. Complexity in construction. 2010.

[5] O. Büyüköztürk, T.Y. Yu, and J.A. Ortega. A methodology for determining complex permittivity of construction materials based on transmission-only coherent, wide-bandwidth free-space measurements. Cement and Concrete Composites, 28(4):349-359, 2006. Non-Destructive Testing.

[6] Ahmed A. Hawam, A.M. El-Kassas, Gouda M. Mahmoud, Ebtisam H. Hasan, and M. Ahmadein. The resolution of analogue measuring devices and its associated uncertainty: An investigation with practical recommendations. Precision Engineering, 71:57-62, 2021.

[7] A. Palacios, M.E. Cong, L.and Navarro, Y. Ding, and C. Barreneche. Thermal conductivity measurement techniques for characterizing thermal energy storage materials - a review. Renewable and Sustainable Energy Reviews, 108:32-52, 2019.

[8] V.S. Ramachandran, Ralph M. Paroli, James J. Beaudoin, and Ana H. Delgado. 1 thermoanalytical techniques. In V.S. Ramachandran, Ralph M. Paroli, James J. Beaudoin, and Ana H. Delgado, editors, Handbook of Thermal Analysis of Construction Materials, pages 1-34. William Andrew Publishing, Norwich, NY, 2002.

[9] A. Petzold and M. Schönlinner. Multi-angle absorption photometry - a new method for the measurement of aerosol light absorption and atmospheric black carbon. Journal of Aerosol Science, 35(4):421-441, 2004.

[10] M. Priel. Incertitudes de mesure et tolérances. Méthodes de mesure, TIB419DUO.(r285), 1999. Technique de l'Ingénieur.

[11] P. De Bièvre. Measurement uncertainty is not synonym of measurement repeatability or measurement reproducibility. Accreditation and Quality Assurance, 13(2):61-62, 2008.

[12] P. Fornasini. The Uncertainty in Physical Measurements: An Introduction to Data Analysis in the Physics Laboratory. Measurements and Uncertainty. Springer, 2008. 289 pp.

[13] AFNOR. Geometrical product specification (gps) - iso code system for tolerances of linear sizes part 1 : basis of tolerances, deviations and fits. Technical Report AFNOR FE119919, Association française de normalisation, 2010.

[14] AFNOR. Geometrical product specifications (gps) - iso code system for tolerances on linear sizes part 2 : tables of standard tolerance classes and limit deviations for holes and shafts. Technical Report AFNOR FE165321, Association française de normalisation, 2010.

[15] ASTM International. Standard specification and temperature-electromotive force (emf) tables for standardized thermocouples. Technical Report ASTM E230-03, American Society for Testing and Materials, 2003.

[16] D. A. H. Fakra, B. R. Alfonso José, N. Mohammad Murad, A. Ny Aina Randriantsoa, and J. C. Gatina. Experimental data and calibration processes to a new and simple device dedicated to the thermo-optical properties of a polycarbonate construction material. Data in Brief, 32:106289, 2020 .

[17] K.F. Carr. Integrating sphere theory and applications part 1: Integrating sphere theory and design. Surface Coatings International, 80(8):380-385, 1997. 
[18] AFNOR. Geometrical product specifications (gps) - geometrical tolerancing - tolerances of form, orientation, location and run-out. Technical report AFNOR E04-552, Association française de normalisation, 2013.

[19] K. Tatsuya, O. Junnosuke, K. Atsuki, and M. Shigenao. Development of guarded hot plate apparatus utilizing peltier module for precise thermal conductivity measurement of insulation materials. International Journal of Heat and Mass Transfer, 91:1157-1166, 2015.

[20] B. Durakovic. Design of experiments application, concepts, examples: State of the art. Periodicals of Engineering and Natural Sciences (PEN), 5(3):421-439, 2017. 


\section{CRediT authorship contribution statement}

Mélanie Delort: Writing - original draft, Software, Formal analysis, Visualization, Validation. Damien Ali Hamada Fakra: Project administration, Writing - review and editing, Funding acquisition, Supervision, Methodology. Bruno Malet-Damour: Conceptualization, Investigation, Data Curation. Jean Claude Gatina: Conceptualization.

\section{Declaration of competing interest}

The authors declare that they have no known competing financial interests or personal relationships that could have appeared to influence the work reported in this paper.

\section{Authors biography:}

Mélanie Delort is a student at the Building Science and Environment (i.e., SBE) Department of the University of La Reunion, France. After obtaining a bachelor's degree in physics (2020), she is pursuing a Master's degree in civil engineering. This article is the result of her Master 1 internship which was an initiation to research funded by the Building Science and Environment department of the Human and Environment Faculty.

Damien Ali Hamada Fakra is a researcher at the SBE Department of the University of La Reunion, France. He obtained a Ph.D. in Buildings Science (2009) and his second Ph.D. in Energy Sciences (2020) at this university. Previously, he received an M.Sc. degree in Mechanical and Energy Engineering at FST-MI University, Morocco. Since 2007, he has participated in 13 European funding projects. He has supervised 35 Master's research internship students and assisted in the supervision of ten Ph.D. students (i.e., coaching thesis).

Bruno Malet-Damour is currently an associate professor in Building Physics at the PIMENT laboratory (https://piment.univ-reunion.fr) of the University of Reunion Island (France). Its research axes in thermal and photometry focus (i) on the study of innovative envelopes based on new materials (from biosourced or waste resources), (ii) the quality of ambiance (multimodal comfort), and (iii) vernacular architecture design. His working method is essentially oriented towards a Galilean sequence (experimenting, modeling, and validating). Since 2019, he is also the project manager of the European Research Project (FEDER) entitled "Phase-change materials: an innovation for tropical buildings.

Jean-Claude Gatina joined the University of La Reunion, France, in 1979 and has been a professor since 1993. His main functions were as follows: Laboratory Director 
(1993-1998), Director of a University Common Service (1993-2001), University Site Director (2001-2008), VP CEVU (2005-2008), and Director of the Training Service (2016-2020). He has directed approximately 40 theses and published approximately 100 articles and papers since 1993. 